\title{
Team Approach for the Management of Amelogenesis Imperfecta
}

\author{
Dr. Sonika Shakya, ${ }^{1}$ Dr. Shaili Pradhan, ${ }^{2}$ Dr. Munna Alam ${ }^{3}$ \\ ${ }^{1}$ Department of Periodontics, KIST Medical College, Lalitpur, Nepal; \\ ${ }^{2}$ Department of Dental Surgery, Bir Hospital, Kathmandu, Nepal; \\ 'Department of Dental Surgery, Shree Birendra Army Hospital, Kathmandu, Nepal.
}

\begin{abstract}
Amelogenesis imperfecta is a developmental disturbance that interferes with normal enamel formation in the absence of a systemic disorder. This case report describes a multidisciplinary team approach for the oral rehabilitation of a young adult patient diagnosed with hypocalcified amelogenesis imperfecta with anterior single tooth crossbite. The specific objectives of this treatment were to enhance esthetics, eliminate tooth sensitivity and restore masticatory function. Treatment included maintenance of gingival health, correction of crossbite, surgical crown lengthening of the maxillary and mandibular posterior teeth and placement of full mouth metal-ceramic crowns. The patient was highly satisfied with the aesthetic and functional outcome of the treatment.

Keywords: Amelogenesis imperfecta; team; management.
\end{abstract}

\section{INTRODUCTION}

Amelogenesis imperfecta (AI) is a developmental disturbance that interferes with normal enamel formation in the absence of a systemic disorder. ${ }^{1}$ Amelogenesis is a two-staged process where a protein rich matrix is initially laid down during the secretary phase, followed by the mineralisation phase where the proteins are replaced by hydroxyapatite crystals. This results in the highly mineralized enamel structure. AI affects the formation of the enamel matrix or the enamel mineralisation process of both the primary and secondary dentition and affects the quantity and quality of the enamel structure and the overall appearance of all or nearly all the teeth. ${ }^{2}$

In its mildest form, AI causes discoloration while in its most severe presentation; the enamel is hypomineralised causing it to be abraded from the teeth shortly after their emergence into the oral cavity. ${ }^{3}$ Apart from enamel defects, AI has also been associated with abnormalities in dental eruption, congenitally missing teeth, anterior open bite, pulpal calcifications, root and crown resorption, hypercementosis, root malformations, and taurodontism. ${ }^{4}$ AI patients may experience compromised chewing function due to tooth sensitivity and the short clinical crowns caused by attrition and/or incomplete eruption. ${ }^{5}$

Aim of the treatment should be to relieve pain or tooth sensitivity, to preserve as much tooth structure as possible

\section{Correspondence:}

Dr. Sonika Shakya

Department of Periodontics,

KIST Medical College, Lalitpur, Nepal.

email: tosonica@gmail.com

\section{Citation}

Shakya S, Pradhan S, Alam M, Team Approach for the Management of Amelogenesis Imperfecta. J Nepal Soc Perio Oral Implantol. 2018;2(1):23-5 while preventing further tooth loss, to maintain mastication and to improve the appearance as this has great psychological impact on the patient. ${ }^{6}$ This case report presents the diagnosis and treatment planning of a 17-year-old girl whose chief concern was unaesthetic appearance of her teeth.

\section{CASE REPORT}

A 17-year-old female patient reported to the Dental Department of Bir Hospital, Kathmandu with the chief complaint of yellow discolouration of teeth and sensitivity of teeth to cold food and drinks. She had discolored teeth since childhood, and her deciduous teeth were similarly discoloured too. Patient reported extreme dissatisfaction with the unaesthetic appearance of her teeth. There was no contributing medical history and family history. On extraoral examination no abnormality was detected. On intra-oral examination, there was generalised yellowish discoloration of all teeth. The incisal edges of upper incisors were chipped off. There was severe attrition on the occlusal surface of all molars. Examination of periodontium revealed chronic generalised gingivitis with calculus deposition and pseudopocket formation (Figures 1 to 5). The orthopantomogram revealed very thin or absent enamel on the occlusal surface of teeth (Figure 6). However, the pulp chamber was within normal limits. Based on history, radiograph and clinical examination a provisional diagnosis of AI-hypocalcified type was made.

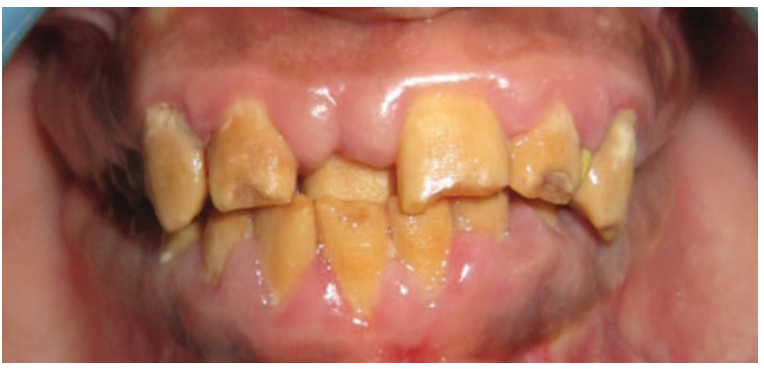

Figure 1: Yellowish discoloration of teeth. 


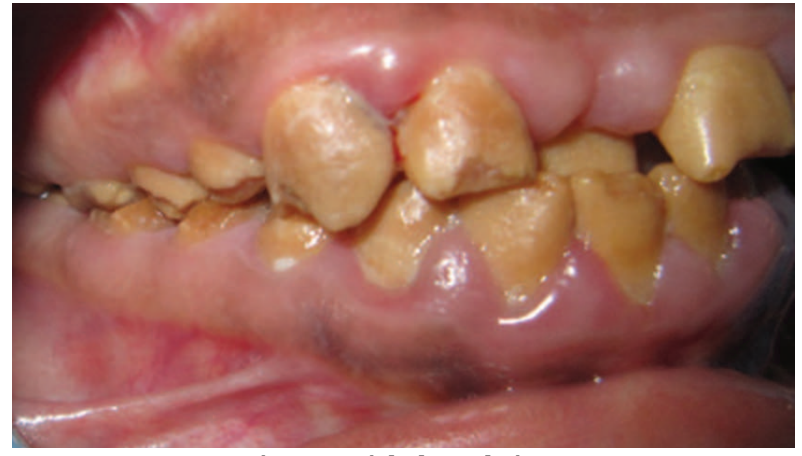

Figure 2: Right buccal view.

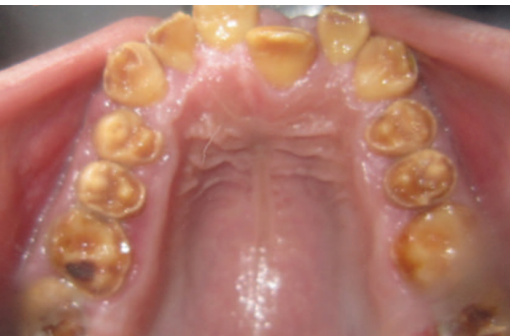

Figure 4: Upper occlusal view.

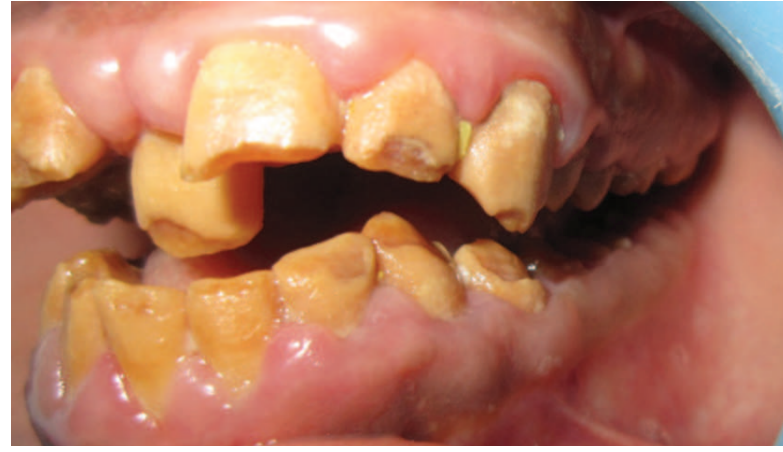

Figure 3: Left buccal view.

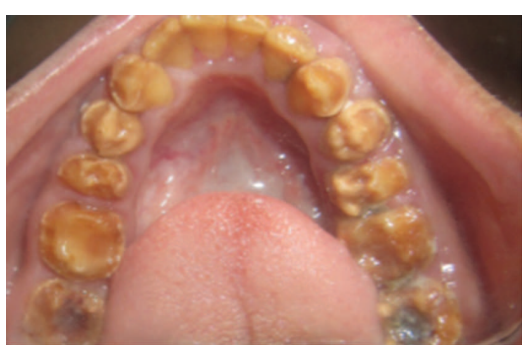

Figure 5: Lower occlusal view.

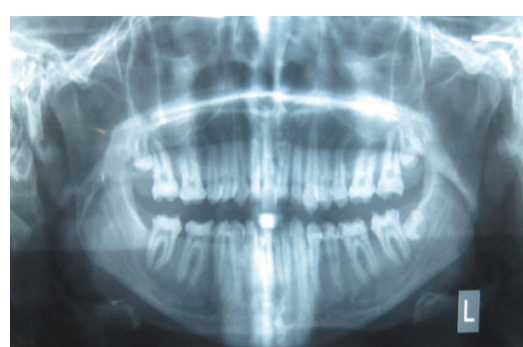

Figure 6: Orthopantomogram.
Diagnostic casts were made. In consultation with the patient full maxillary and mandibular rehabilitation with porcelain fused metal (PFM) crowns extending to the second molars was considered to be the best therapeutic option. As the clinical crowns were inadequate for the placement of the PFM crowns, surgical crown lengthening was planned.

There was generalised gingival overgrowth which almost covered the occlusal third of the crown especially in the upper and lower molars. The patient had poor oral hygiene due to sensitivity during brushing. By looking at the gingival status the first phase of treatment included patient motivation and education and reinforcement on brushing techniques. Scaling was done followed by rootplaning. Once the gingival health

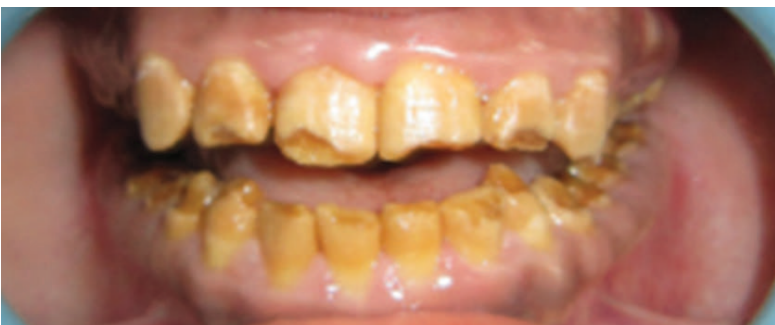

Figure 7: After completion of scaling \& root planing \& orthodontic treatment.

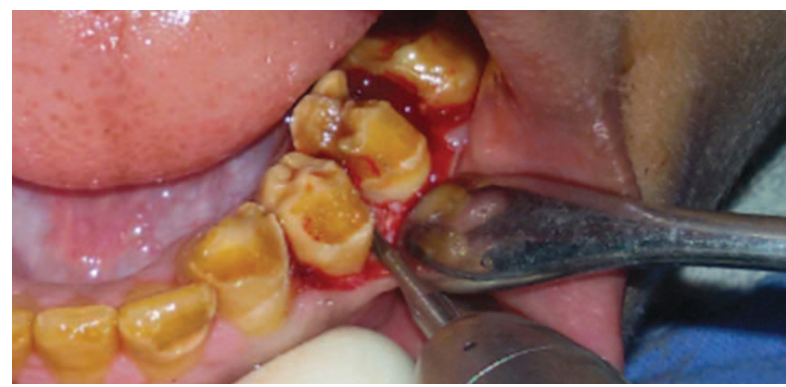

Figure 9: Osteotomy. was improved orthodontic treatment was started to correct the crossbite with respect to 11 (Figure 7).

The next phase of treatment included restoration of carious lesions and root canal treatment where required. As planned earlier, surgical crown lengthening was done in all the posterior teeth. In the first and second quadrant, external bevel gingivectomy was planned due to the presence of pseudopockets (Figure 8).

In the third quadrant, internal bevel gingivectomy with osteotomy was done (Figure 9).

In the fourth quadrant, internal bevel gingivectomy along with distal wedge surgery was done (Figure 10). Provisional

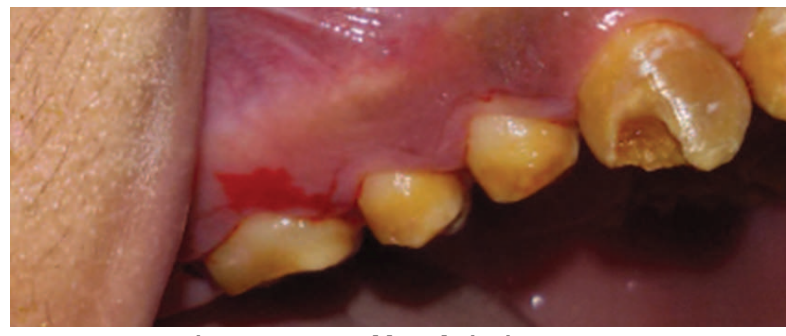

Figure 8: External bevel gingivectomy.

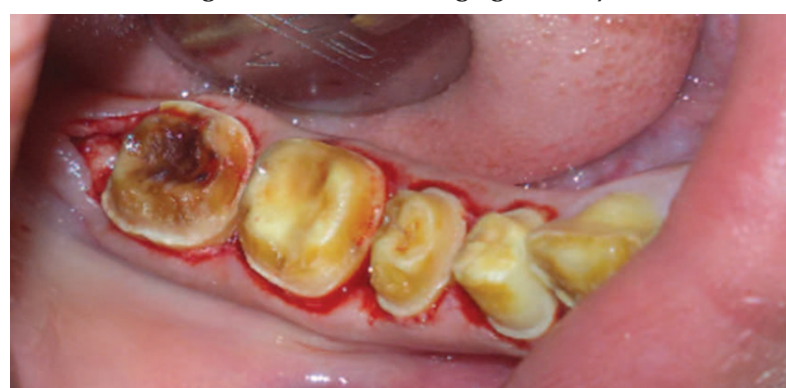

Figure 10: Distal wedge surgery. 


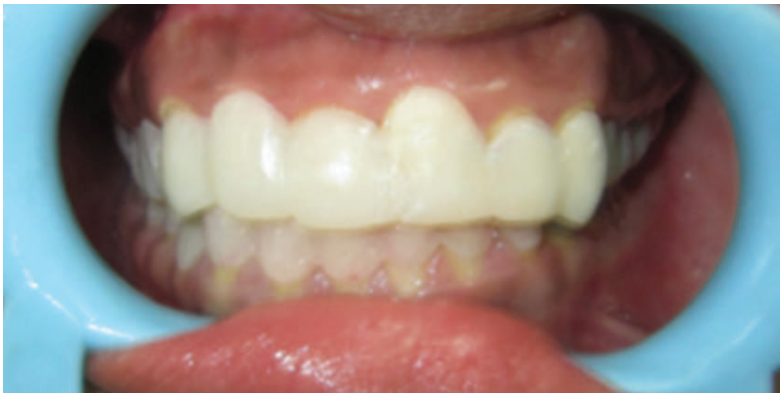

Figure 11: Provisional restoration.

restoration was given in all the quadrants (Figure 11). After six weeks tooth preparation for porcelain fused to metal crown restoration was started for all teeth and final restoration was given (Figure 12).

\section{DISCUSSION}

Clinical appearances of AI may vary among individuals, from discoloration of teeth (yellow, brown or gray) to generalized areas of exposed dentine and enamel pitting. Increased susceptibility to plaque accumulation, caries, hypersensitivity and loss of vertical dimensions are the common clinical presentations. ${ }^{6}$ Management of patients with AI provides great challenges to clinicians both from a functional and an esthetic perspective. ${ }^{7}$ Treatment objectives may vary depending on the age of the patient, socioeconomic status of the patient, severity of the disorder and the intraoral status at the time of treatment planning. ${ }^{8}$ The primary goal of the treatment should address each concern as it presents but with an overall comprehensive plan that outlines the anticipated future treatment needs. ${ }^{9}$ Hence a multidisciplinary team approach is very much essential to treat patients with AI.

In the present case, the patient's chief complaint was her

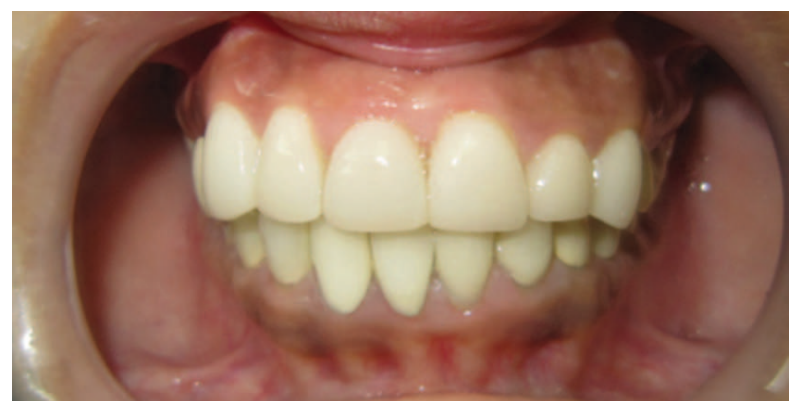

Figure 12: Final restoration.

poor esthetics. All of her posterior teeth were attrited causing severe sensitivity. So, full coverage porcelain fused to metal crowns were planned. Due to severe sensitivity her oral hygiene was poor leading to gingival overgrowth almost covering the already short clinical crowns. We all know that periodontal health is the cornerstone of any successful restorative procedure. Therefore, the correct handling of the periodontal tissues during restoration of the tooth is important for the long term success of the restoration.10 Thus, it is necessary to prepare periodontal tissues properly before restorative treatment to ensure good form, function, and esthetics of masticatory apparatus and patient comfort. So, scaling and root planing was performed and surgical crown lengthening was done where required to maintain the biologic width during restoration.

Hence, early treatment of patient with AI is necessary to prevent progressive damage of dentition and the psychological impact of this condition on the patient. Unfavorable effects on aesthetic, function, occlusion necessitate different approaches from traditional dental treatments. So, by systematic and sequential interdisciplinary treatment approach patient's esthetic and functional requirements can be fulfilled.

\section{REFERENCES}

1. White SC, Pharoah MJ. Dental anomalies. In: Oral Radiology: Principles and Interpretation. 5th ed. St. Louis, MO: Mosby/Elsevier; 2009:307-11.

2. Lindunger A, Smedberg J I. A retrospective study of the prosthodontic management of patients with amelogenesis imperfecta. Int J Prosthodont. 2005;18:189-94

3. Chanmougananda SC, Ashokan KA, Ashokan SC, Bojan AB, Ganesh RM. Literature review of amelogenesis imperfecta with case report. J Indian Acad Oral Med Radio. 2012;24:83-7.

4. de Souza JF, Fragelli CM, Paschoal MA, Campos EA, Cunha LF, Losso EM, et al. Noninvasive and multidisciplinary approach to the functional and esthetic rehabilitation of amelogenesis imperfecta: A pediatric case report. Case Rep Dent. 2014;12;71-5.

5. Chaudhary M, Dixit S, Singh A, Kunte S. Amelogenesis imperfecta: Report of a case and review of literature. J Oral Maxillofac Pathol. 2009;13:70-7.

6. Seow WK. Clinical diagnosis and management strategies of amelogenesis imperfecta variants. Pediatr Dent. 1993;15:384-93.

7. Ayers KM, Drummond BK, Harding WJ, Salis SG, Liston PN. Amelogenesis imperfect - multidisciplinary management from eruption to adulthood. Review and case report. NZ Dent J. 2004;100:101-4.

8. Gokce K, Canpolat C, Ozel E. Restoring function and esthtetics in patient with amelogenesis imperfecta: A case report. J Contemp Dent Pract. 2007;8:95-101.

9. American Academy on Pediatric Dentistry Council on Clinical Affairs. Guideline on oral health care/dental management of heritable dental developmental anomalies. Pediatr Dent. 2008-2009;30(7):196-201.

10. Planciunas L, Puriene A, Mackeviciene G. Surgical lengthening of the clinical tooth crown. Stomatologica. 2006;8:88-95. 